\title{
Uniportal video-assisted thoracic surgery: Pelotas-RS, Brazil, 2018
}

\author{
Otávio Leite Gastal' ${ }^{1}$, Ricardo Lanzetta Haack², Diego Gonzales Rivas ${ }^{3}$, Laura Nunes Rotta ${ }^{4}$ Izabel de \\ Oliveira Karam ${ }^{5}$
}

${ }^{1}$ Department of Surgery, Federal and Catholic Universities of Pelotas, Thoracic Surgery Unit, ${ }^{2}$ Department of Surgery, Federal and Catholic Universities of Pelotas, Oncologic Surgery Unit, Beneficência Portuguesa Hospital, Pelotas, Brazil; ${ }^{3}$ Department of Thoracic Surgery, Coruña University Hospital and Minimally Invasive Thoracic Surgery Unit (UCTMI), Coruña, Spain; ${ }^{4}$ Medical student at the Catholic University of Pelotas, Pelotas, Brazil; ${ }^{5}$ Medical student at the Federal University of Pelotas, Pelotas, Brazil

Correspondence to: Otávio Leite Gastal. Department of Surgery, Federal and Catholic Universities of Pelotas, Thoracic Surgery Unit, Beneficência Portuguesa Hospital, Pelotas, Brazil. Email: otaviogastal@gmail.com.

\begin{abstract}
Video-assisted thoracic surgery (VATS) has proved to be the gold-standard for biopsing and treating both benign and malignant diseases, and in the past few years this approach has been improved to a unique incision. On July 2018 Dr. Otávio Leite Gastal attended the "Uniportal VATS Training Program" at Shanghai's Hospital, China and Dr. Diego Gonzales Rivas, its coordinator, was invited to a master class and live surgery procedures in southern Brazil. The event took place at the Beneficência Portuguesa Hospital, Pelotas, RS, Brazil and consisted on case presentations, followed by surgical procedures and concluded with an evening master class. After the event, the uniportal VATS procedures became a first-line technique at our center, reinforcing the importance of Dr. Diego Rivas' tutoring and effort.
\end{abstract}

Keywords: Uniportal video-assisted thoracic surgery (Uniportal VATS); lobectomy; segmentectomy; thoracic surgery; minimally invasive

Received: 01 November 2018; Accepted: 14 November 2018; Published: 04 December 2018.

doi: $10.21037 /$ jovs.2018.11.05

View this article at: http://dx.doi.org/10.21037/jovs.2018.11.05

Video-assisted thoracic surgery (VATS) has enabled lobectomies in Brazil since 1992 (1), however this type of surgery has faced several obstacles, such as proper training for surgeons and assistants in addition to equipment acquisition (2,3). Although videothoracoscopy has proved to be gold-standard for biopsing and treating both benign and malignant diseases, even in major procedures $(1,4,5)$, the aforementioned obstacles have hampered its development, preventing it from spreading faster in the country (1).

In the last few years, this technique has been refined from a multiportal approach to a minimal invasive process, using only one incision of less than $4 \mathrm{~cm}(1,2)$. This approach has improved the advantages of minimally invasive operations, maintaining effectiveness in many different procedures $(3,5)$, including wedge resection, drainage for empyema and pleural effusion, resection of mediastinal tumors, segmentectomies and lobectomies (4).
Patients undergoing the uniportal VATS technique recognize its benefits, experiencing less pain in postoperative and faster recoveries (6).

In Brazil, this new technique was introduced in 2016 in São Paulo, by its own idealizer Dr. Diego Gonzalez Rivas (1). More recently, the city of Pelotas, in southern Brazil, had the opportunity to host this world-class surgeon and participate of his worldwide effort, the so-called "Uniportal VATS Training Program" (7) in 2018. This SouthernBrazilian medium-sized town represents a health center for its region and is responsible for 845 thousand people from 22 nearby cities, acting as a reference endpoint for many medical specialities, among them, thoracic surgery (8).

The population of Pelotas is around 330,000 people (8), supported by seven private and public hospitals (9). The Beneficência Portuguesa Hospital is a philanthropic institution, located in the center of the city, offering 255 hospital beds 


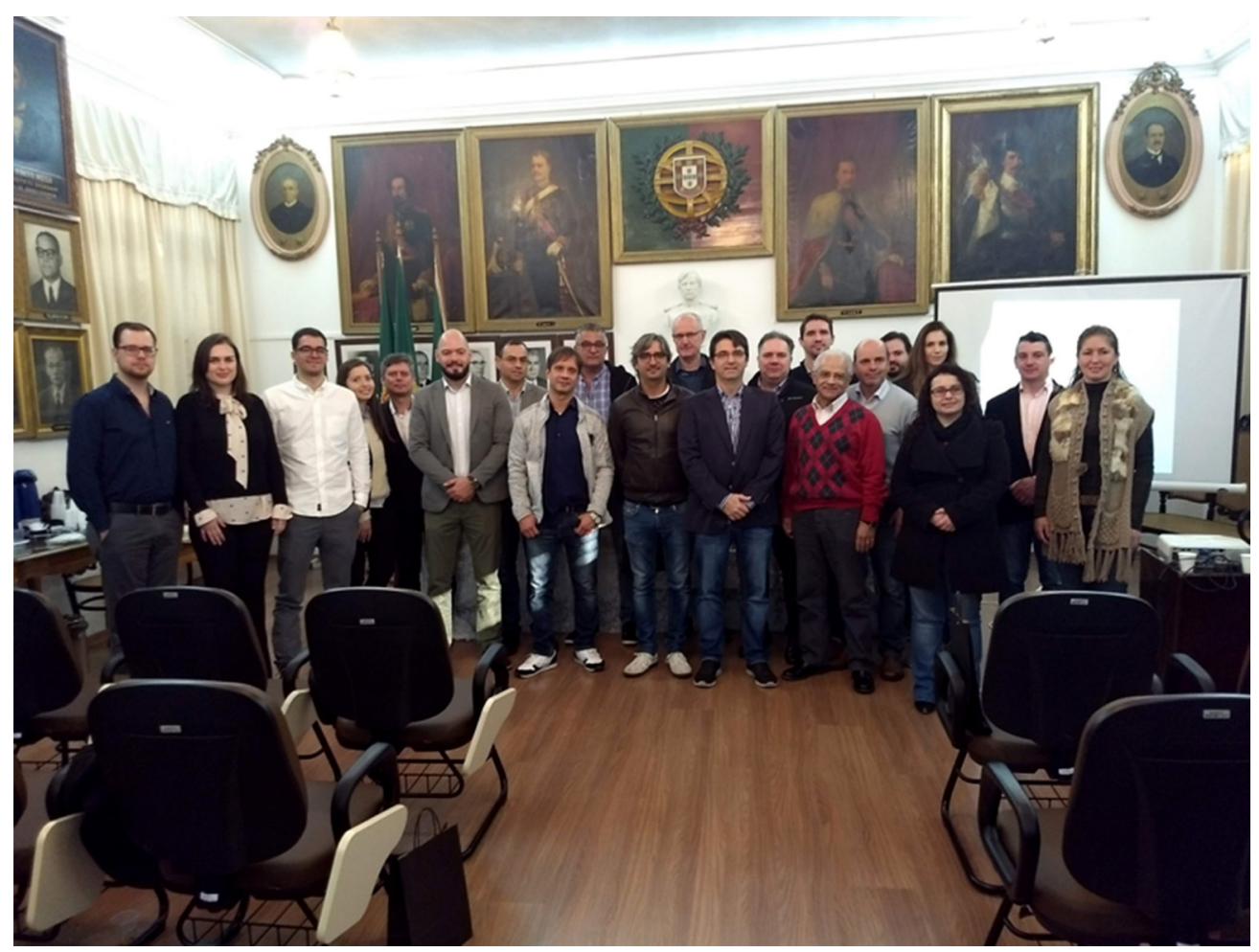

Figure 1 Meeting at the "honor room" with all participants.

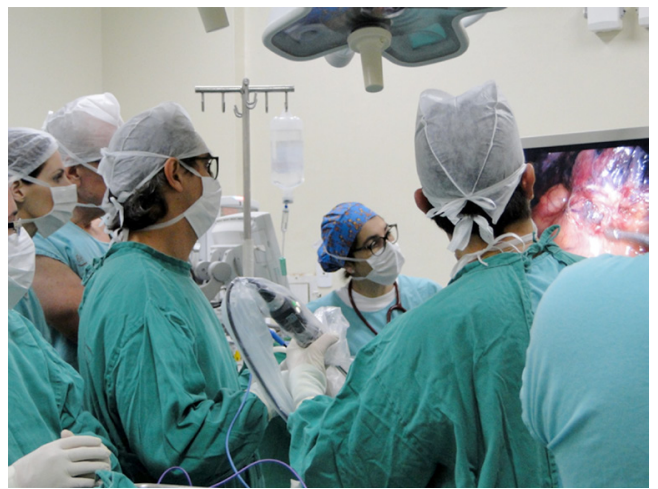

Figure 2 Local surgeons attending to surgical procedures performed by Dr. Rivas.

for many specialities, including intensive care and cardiology intensive care units (10). After Dr. Otávio Leite Gastal attended to lectures and training program at the Shanghai's Hospital, Dr. Diego Rivas offered to perform and teach local colleagues the uniportal VATS approach in this hospital surgery center. It was also his intention to demonstrate the feasibility of its implementation at this institution.

The course took place at the Beneficencia Portuguesa
Hospital of Pelotas and consisted in a full day of uniportal VATS. Early on the morning of August 17th, 2018 the whole group met at the "honor room" of the Hospital to welcome the participants of the event. Mr. José Leal Serra, the hospital's president, introduced Dr. Diego Rivas from Spain and Dr. Rodrigo Sardenberg from São Paulo to the participants of the event (Figure 1).

Afterwards, two cases were presented by Dr. Otávio Leite Gastal prior to the surgical procedures and the whole group was invited to attend live surgeries performed by Dr. Gonzalez-Rivas (Figure 2). The first case was a S3 segmentectomy due to ground-glass nodular opacity on a female patient. The second patient was a male patient with cavitated epidermoid carcinoma treated with a left-inferior lobectomy. Patients were placed in a lateral decubitus position, the incisions were made in the fifth intercostal space, and no wound retractor was placed. Both operations were completed without any complications. Informed consent was received from all patients.

\section{CASE 1}

A 76-year-old white female, retired (previously a housewife), 

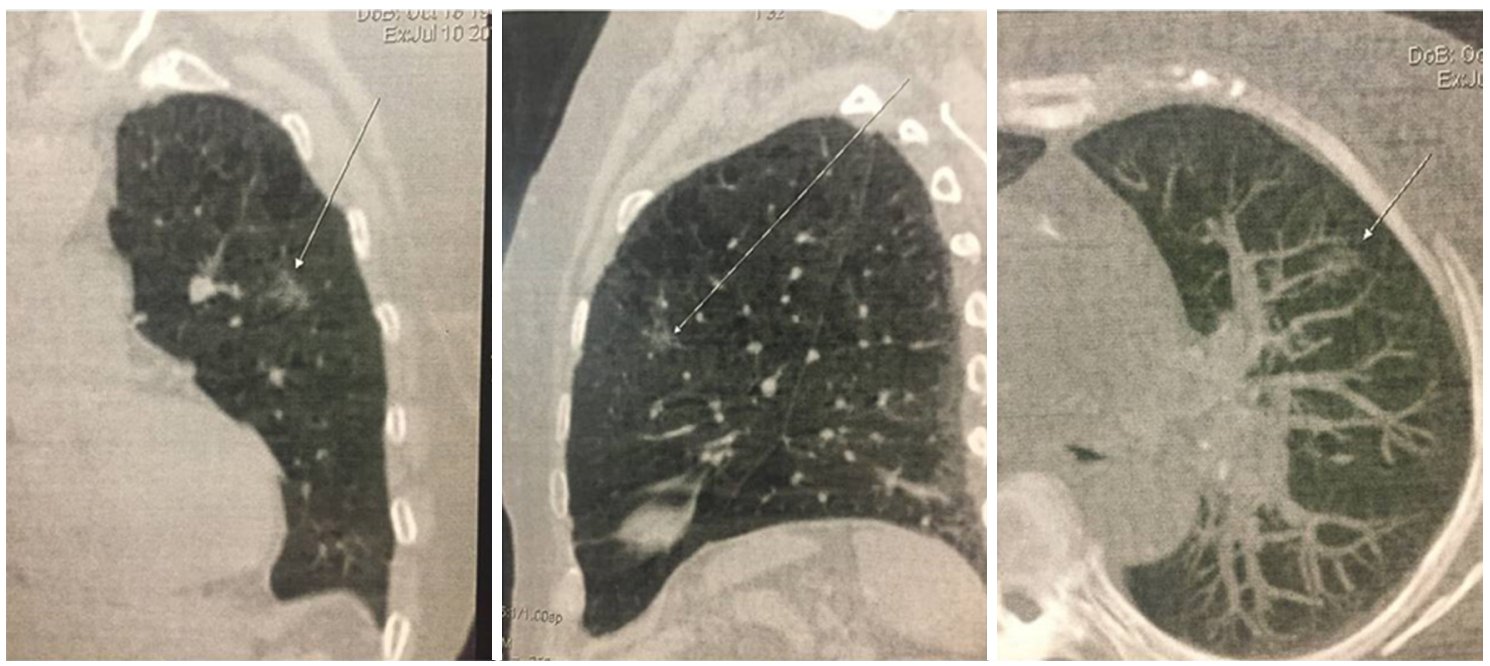

Figure 3 Thorax CT scan demonstrating nodular ground-glass opacity of $1.2 \mathrm{~cm}$, indicated by the sign. CT, computed tomography.

who had smoked an average of 15 cigarettes a day for almost 60 years. Thirty days prior to the surgery, the patient presented with cough, associated with a few expectoration and medium effort dyspnea. History of surveillance with a pneumologist for 11 years, occasionally using beta-2 adrenergic agonist. Discreet peripheral cyanosis, diffusely decreased vesicular breathing sounds, normal cardiac auscultation, oxygen saturation of $95 \%$ and blood pressure of $130 \times 80 \mathrm{mmHg}$.

Echocardiogram indicating left-ventricular diastolic dysfunction, impaired relaxation pattern and discreet aortic valve dysfunction. Spirometry denoting mild obstructive respiratory disturb, with no response to bronchodilator. Thorax CT scan (Figure 3) showing nodular groundglass opacity of $1.2 \mathrm{~cm}$, with spiculated surroundings, of uncertain nature and evolutive potential.

\section{CASE 2}

A 63-year-old white male, active as a real estate agent, who started smoking at the age of 12 years old and had stopped smoking 4 months prior to the surgery. The patient had presented poorly productive cough for the last 10 months. Diabetic in medical therapy and adequate diet. Normal clinical exam.

Thorax CT scan (Figure 4) pointed excavated lesion of
$4.8 \times 3.6 \mathrm{~cm}$. Fibrobronchoscopy and bronchial brushing and washing were inconclusive. At the time, the patient received antibiotics showing partial improvement.

"Lunch with the professor" was offered in a very pleasant social club by the Pelotas river and a typical barbecue was served. In evening, a Master Class by Dr. Gonzalez-Rivas was presented highlighting the benefits and applications of uniportal VATS surgery (Figure 5). Fifteen professionals from different cities of the whole state of Rio Grande do Sul were invited for the whole day program. The master class was opened for an additional group of pulmonologists, thoracic surgeons and oncologists from the community.

Despite being considered a new technique, it has been introduced in many other surgical centers before, its safety and efficacy have been scientifically proved, allowing professionals to feel safe to perform the uniportal VATS $(3,4,11)$. It offers many substantial advantages, among them, shorter chest drainage duration, minimal postoperative hospital stay and less painkillers in this period $(11,12)$.

Due to this process, the uniportal VATS has become a first-line technique for even complex surgeries, such as advanced lung cancer, at our center. The tutoring of Dr. Diego was indispensable to speed up the learning process, leading to surgeons presenting proper skills to perform this type of surgery safely and with confidence even when faced with more difficult cases. 

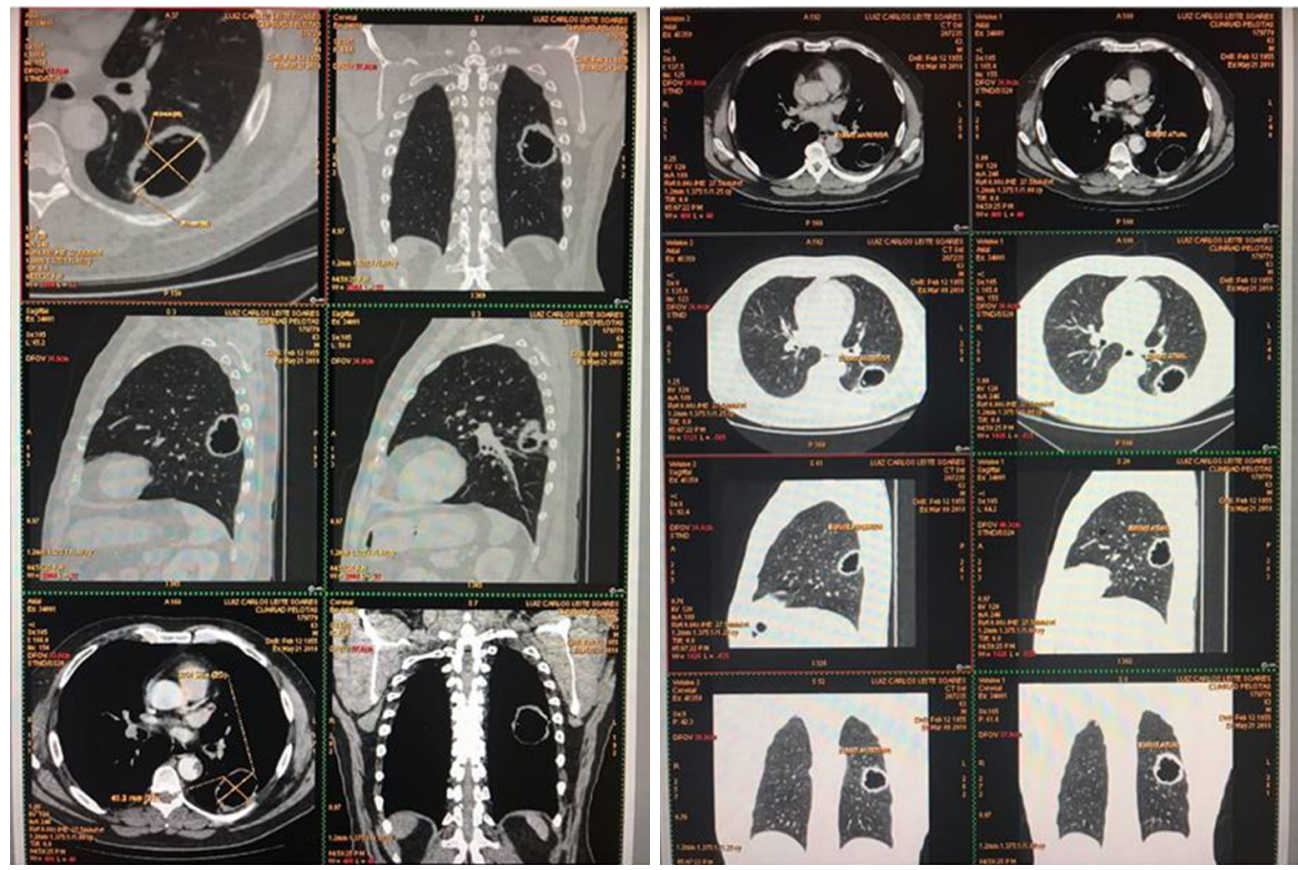

Figure 4 Thorax CT pointing excavated lesion in left lung, measuring $4.8 \times 3.6 \mathrm{~cm}$. CT, computed tomography.

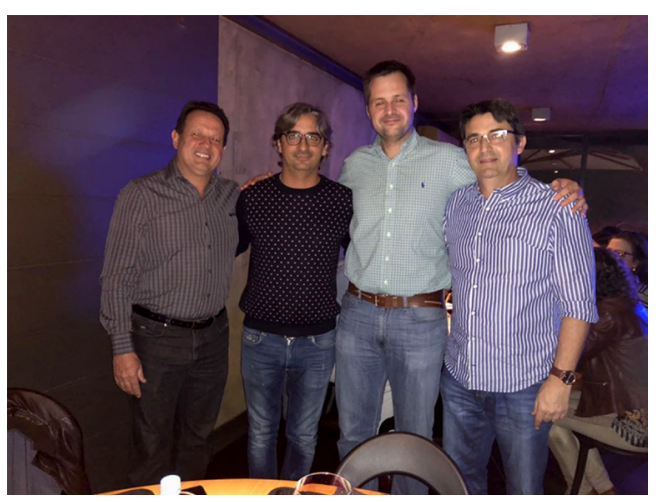

Figure 5 Eduardo Rotta, Diego Rivas, Ricardo Haack and Otávio Gastal, after the Master Class presentation.

\section{Acknowledgments}

Funding: None.

\section{Footnote}

Provenance and Peer Review: This article was commissioned by the editorial office, Fournal of Visualized Surgery for the series "Teaching Uniportal VATS". The article has undergone external peer review.
Conflicts of Interest: All authors have completed the ICMJE uniform disclosure form (available at http://dx.doi. org/10.21037/jovs.2018.11.05). The series "Teaching Uniportal VATS" was commissioned by the editorial office without any funding or sponsorship. DGR served as the unpaid Guest Editor of the series and serves as an unpaid associate editor-in-chief of Fournal of Visualized Surgery. The authors have no other conflicts of interest to declare.

Ethical Statement: The authors are accountable for all aspects of the work in ensuring that questions related to the accuracy or integrity of any part of the work are appropriately investigated and resolved. All procedures performed in studies involving human participants were in accordance with the Helsinki Declaration (as revised in 2013). Written informed consent was obtained from the patient for publication of this manuscript and any accompanying images.

Open Access Statement: This is an Open Access article distributed in accordance with the Creative Commons Attribution-NonCommercial-NoDerivs 4.0 International License (CC BY-NC-ND 4.0), which permits the noncommercial replication and distribution of the article with 
the strict proviso that no changes or edits are made and the original work is properly cited (including links to both the formal publication through the relevant DOI and the license). See: https://creativecommons.org/licenses/by-nc-nd/4.0/.

\section{References}

1. Sardenberg RA, Gonzalez-Rivas D. Uniportal video assisted thoracic surgery right main sparing lung bronchial resection: live surgery in Sao Paulo. J Vis Surg 2017;3:166.

2. Schewitz I. Uniportal lobectomy in Africa: a beginning. J Vis Surg 2016;2:54.

3. Yu PS, Ng CS. Recent developments in uniportal VATS in Asia and beyond. J Vis Surg 2018;4:184.

4. Guido Guerrero W, Gonzalez-Rivas D, Yang Y, Li W. The evolution of uniportal video assisted thoracic surgery in Costa Rica. J Vis Surg 2016;2:91.

5. Gonzalez-Rivas D, Fieira E, Delgado M, et al. Uniportal video-assisted thoracoscopic lobectomy. J Thorac Dis 2013;5:S234-45.

doi: 10.21037 /jovs.2018.11.05

Cite this article as: Gastal OL, Haack RL, Rivas DG, Rotta LN, Karam IO. Uniportal video-assisted thoracic surgery: Pelotas-RS, Brazil, 2018. J Vis Surg 2018;4:243.
6. Sihoe AD. Reasons not to perform uniportal VATS lobectomy. J Thorac Dis 2016;8:S333-43.

7. Sociedade Brasileira de Cirurgia Torácica. Uniportal VATS Training Program. Available online: https://www.sbct.org. br/uniportal-vats-training-program

8. Instituto Brasileiro de Geografia e Estatística. Available online: https://www.ibge.gov.br/

9. Cadastro Nacional de Estabelecimentos em Saúde. Available online: http://cnes.datasus.gov.br/pages/ estabelecimentos/consulta.jsp

10. Hospital Beneficência Portuguesa. Available online: http:// www.beneficencia-pelotas.com.br

11. Todd Demmy. Elisabeth Dexter. 2018. UpToDate: Overview of minimally invasive thoracic surgery. Available online: https://www.uptodate.com/contents/overviewof-minimally-invasive-thoracic-surgery? search=VATS \&source=search_result\&selectedTitle=1 122\&usage_ type $=$ default\&display_rank=1\#H19002712

12. Ismail $M$, Helmig $M$, Swierzy $M$, et al. Uniportal VATS: the first German experience. J Thorac Dis 2014;6:S650-5. 